$>$ Le cancer de la vessie se présente sous deux formes différentes: la forme superficielle et la forme infiltrante. La forme superficielle se caractérise par une fréquence élevée de pertes (totales ou partielles) du chromosome 9, notamment du bras long. Ces observations suggèrent la présence de gènes suppresseurs de tumeur (GST) dans cette région. Nous présentons le gène PATCHED, localisé en position 9q22.3, déjà impliqué dans le carcinome basocellulaire de la peau, comme un GST potentiel dans le cancer superficiel de la vessie. <

\section{La voie de signalisation PATCHED/Sonic Hedgehog dans le cancer superficiel de la vessie}

Hélène LaRue, Maryse Simoneau, Tahar 0. Aboulkassim, Patricia Lemieux, Johanne Girard, Sahar Hamed, Hélène Hovington, Lucie Jeannotte, Yves Fradet
Le cancer de la vessie se situe au cinquième rang, en terme d'incidence, dans les sociétés occidentales. Son étiologie est principalement associée à la présence de produits carcinogènes dans l'environnement, notamment ceux de la fumée de cigarette et des émanations d'origine industrielle. Lors du diagnostic initial, 75 à $80 \%$ des patients présentent un cancer superficiel papillaire (stade TA ou $\mathrm{Tl}$ ) et pour plus de $60 \%$ de ces patients, l'évolution sera marquée par des récidives parfois fréquentes avec progression vers l'infiltration musculaire dans $10 \%$ des cas. Le reste des patients présentent un cancer d'emblée infiltrant (stade T2-T4) dérivant d'un carcinome in situ (CIS) de grade élevé et d'une dysplasie. Bien que la mortalité associée au cancer de la vessie soit due aux tumeurs infiltrantes, la morbidité provient principalement des tumeurs superficielles récidivantes et de leur traitement par résection transurétrale et chimiothérapie ou immunothérapie intravésicale.

\section{Anomalies du chromosome 9 dans le cancer de la vessie}

Les différentes expressions cliniques des cancers de la vessie ont pu être associées à des voies moléculaires distinctes [1]. Alors que l'on retrouve souvent des

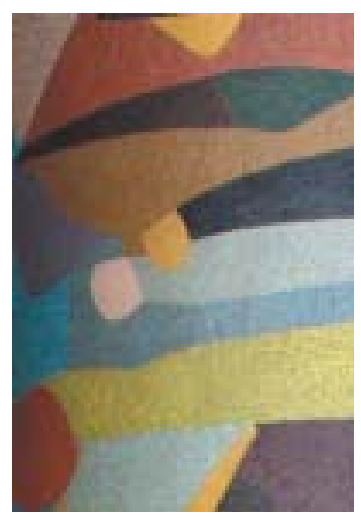

mutations du gène $p 53$
Centre de recherche en cancéro-

logie de l'Université Laval, CHUQ-L'Hôtel-Dieu de Québec,

10 , rue MacMahon, GIR 2J6 Québec, Canada.

yves.fradet@crhdq.ulaval.ca dans les CIS et les

tumeurs infiltrantes, des anomalies du chromosome 9 sont fréquentes dans les tumeurs superficielles. Diverses études ont montré des pertes d'hétérozygotie ( $\mathrm{LOH}$, loss of heterozygosity) sur ce chromosome dans plus de la moitié des cancers de vessie et il s'agit souvent de l'unique altération génétique observée dans les tumeurs à un stade peu avancé. Ces données suggèrent la présence d'un ou plusieurs gènes suppresseur de tumeur (GST) sur le chromosome 9, intervenant dans les phases précoces de la cancérogenèse vésicale. Plusieurs études ont suggéré que les locus en $9 p 22-23,9 p 21-22,9 p 11-13$, $9 q 12-13,9 q 21-22,9 q 31,9 q 33$ et $9 q 34$ seraient susceptibles de porter des GST [1-4]. Lors d'une recherche de $\mathrm{LOH}$ au niveau de 28 marqueurs microsatellites du chromosome 9 dans 139 tumeurs initiales de stade TA ou Tl, nous avons répertorié 67 tumeurs (48\%) présentant une ou plusieurs $\mathrm{LOH}$ sur le chromosome 9, ce qui nous a conduit à proposer 4 locus au niveau du bras $9 q, 9 q 22.3$, $9 q 31-32,9 q 33$ et $9 q 34$ comme étant des sites potentiels de GST [2]. Quelques GST candidats ont été associés à certains locus du chromosome 9 tels CDKN2A à $9 \mathrm{p} 21$, 
PATCHED (PTC) à 9q12-31, DBCCR1 à 9q32-33 et TSCl à $9 q 34[1,4]$. La présence de LOH sur le chromosome 9 a de plus été associée à un risque élevé de récidive [5] et nous avons observé que des patients porteurs des tumeurs superficielles contenant des délétions dans les régions $9 p t r-p 22,9 q 22.3,9 q 33$ et $9 q 34$ avaient une survie sans récidive significativement plus courte que des patients dont les tumeurs étaient exemptes de délétions (Figure 1) [6], ce qui est compatible avec la présence de GST au niveau de ces locus.

Dans le but d'identifier le GST de la région 9q22.3, nous avons poursuivi notre cartographie du chromosome 9 en analysant 11 marqueurs microsatellites additionnels dans cette région, sur 139 tumeurs de stade peu avancé [7]. Cette étude a permis de définir une région minimale de délétion de $0,5 \mathrm{Mb}$ incluant, le gène PTC, candidat GST précédemment proposé. Dans $46 \%$ de l'ensemble des tumeurs analysées, nous avons observé la perte d'un marqueur microsatellite situé dans l'intron 1 du gène PTC [7]. Ces données nous ont conduit à proposer que PTC soit effectivement un GST candidat de la région 9q22.3 dont la délétion serait un événement précoce de la cancérogenèse vésicale.

\section{Voie de signalisation PTC/Hedgehog chez la drosophile}

Le gène PTC humain est l'orthologue du gène ptc de la drosophile, d'abord identifié comme étant un gène important au cours du développement embryonnaire [8]. Chez la mouche, le gène ptc joue un rôle dans l'élaboration de l'axe antéro-postérieur des embryons et dans la polarité des segments. II code pour une protéine possédant 12 domaines transmembranaires, deux boucles extracellulaires et un domaine intracellulaire. Cette protéine agit comme récepteur de la protéine sécrétée hedgehog (hh) (Figure 2). En l'absence de hh, ptc inhibe la voie de signalisation en formant un complexe avec une seconde protéine membranaire, smoothened (smo), et en réprimant l'activité de cette dernière. D'autres protéines engagées dans cette voie de signalisation ont également été décrites chez la drosophile, la protéine costal-2 (cos-2), la sérine-thréonine kinase fused (fu), le suppresseur de fused (su[fu]) et le facteur de transcription à doigts de zinc cubitus interruptus (ci). Ces protéines forment un complexe cytoplasmique lié aux microtubules. Lorsque la voie est inactive, ci subit un clivage qui libère un fragment amino-termi- 
nal de 75 kDa. Ce fragment pénètre dans le noyau où il agit comme répresseur transcriptionnel. La liaison de hh à ptc libère smo, ce qui entraîne une dissociation du complexe cytoplasmique et l'inhibition du clivage de ci. La protéine ci intacte subit alors une maturation qui la transforme en un activateur transcriptionnel labile de divers gènes dont $p t c$, decapentaplegic $(d p p)$, un membre de la famille TGF $\beta$, et wingless ( $w g)$, orthologue des gènes Wnt des vertébrés [9].

\section{Voie de signalisation PTC/SHH chez les vertébrés}

Des homologues de plusieurs membres de cette voie ont été identifiés chez les vertébrés où celle-ci, bien que présentant un niveau plus élevé de complexité, semble fonctionner de façon similaire à celle de la drosophile $[10,11]$. Deux homologues de ptc, PTCl et PTC2, ont été décrits comme pouvant tous les deux interagir avec $\mathrm{HH}$ et smo $[9,11]$. Cependant, bien que PTCl, dont le gène est situé sur le chromosome 9 , soit bien caractérisé, on connaît peu le rôle de PTC2. Trois homologues de hh ont également été identifiés, Sonic ( $\mathrm{SHH})$, Indian $(\mathrm{IHH})$ et Desert (DHH) Hedgehog [9]. Ces trois ligands utilisent la même voie de signalisation. Un seul homologue de smo a été identifié jusqu'à présent, mais trois facteurs de transcription à doigts de zinc, GLI-1, GLI-2 et GLI-3 occupent la position de ci $[8,12]$. GLI-1 semble agir essentiellement comme activateur et serait le principal effecteur transcriptionnel de cette voie tandis que GLI-2 et GLI-3 possèdent des domaines activateurs et répresseurs de la transcription et sont modifiées par clivage protéolytique comme ci. Cependant, des incertitudes importantes subsistent quant à la transduction du signal SHH par les protéines GLI et la plupart des études suggèrent un équilibre complexe entre des fonctions divergentes. Une seule protéine cytoplasmique agissant en amont de GLI, l'homologue de su(fu), a été identifiée dans des cellules humaines [11].

\section{Voie de signalisation PTC/SHH et prolifération cellulaire}

Bien que les mécanismes sous-jacents au fonctionnement de la voie PTC/SHH demeurent encore mal compris, il apparaît que son activation se traduit principalement par une prolifération accrue. Le haut degré de conservation de cette voie chez la drosophile et les vertébrés permet de supposer que l'activation de la prolifération implique la participation des membres des familles TGF $\beta$ et WNT, ces dernières possédant un large spectre de fonctions. De plus, des données récentes ont permis d'établir un lien direct entre l'activation par hh et l'augmentation de la transcription des cyclines $D$ et $\varepsilon$, pro- téines directement engagées dans l'activation du cycle cellulaire [13]. Enfin, la transfection de cellules rénales de rat avec le gène Gli-l entraîne des modifications du profil génique compatibles avec un accroissement de la prolifération par des voies diverses comme l'augmentation de la division cellulaire, la diminution de l'adhérence cellulaire et de l'apoptose. Des sites de reconnaissance de la protéine Gli-1 dans le promoteur d'au moins quatre des gènes modulés (cycline D2, IGFBP-6, osteopontine et plakoglobine) ont pu être identifiés [14].

\section{Voie de signalisation PTC/SHH et cancer}

Le rôle du gène PTC en tant que GST a été initialement démontré pour le carcinome basocellulaire de la peau [9]. En effet, des mutations du gène PTC sont fréquem-

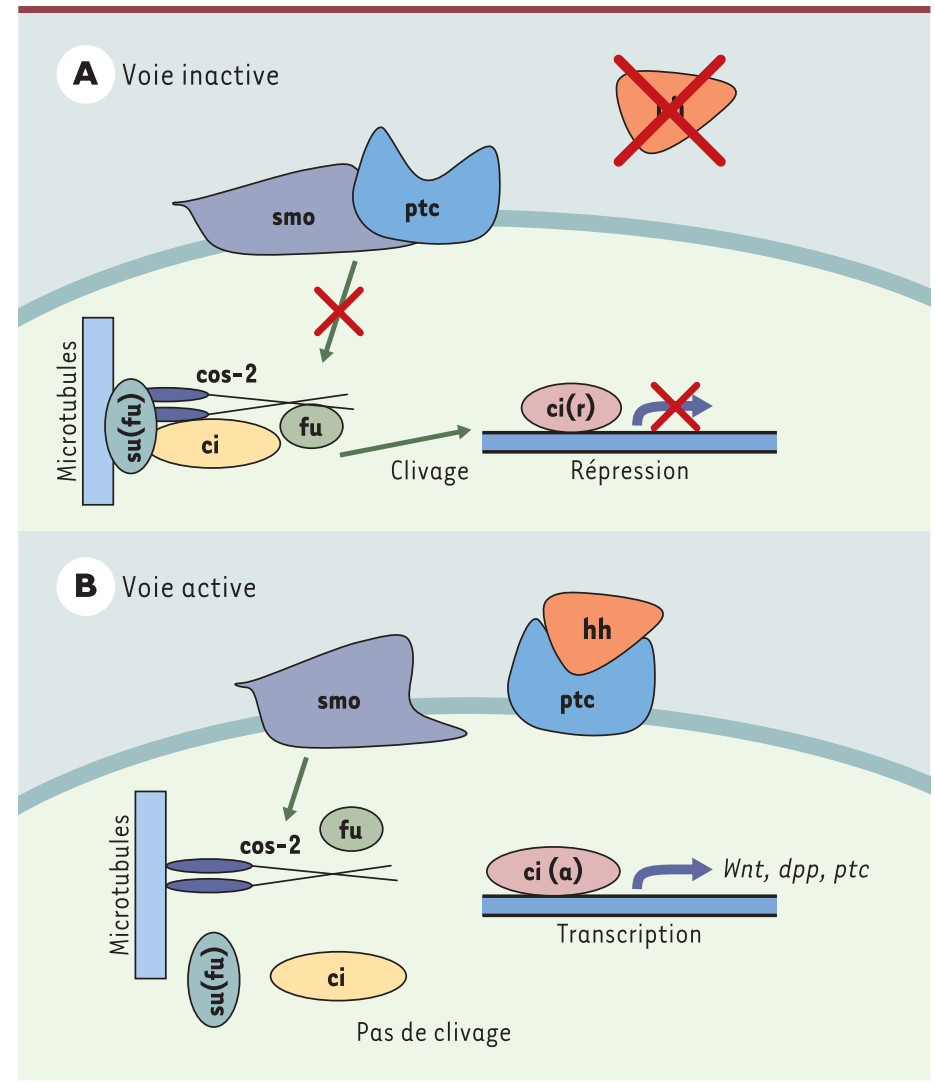

Figure 2. Modèle proposé pour la voie de signalisation ptc/hh chez la drosophile, d'après les données les plus récentes. $A$. La voie est inactive lorsque ptc (Patched) se lie à la protéine smo (smoothered) et en inhibe l'activité. Un complexe de protéines cytoplasmiques liées aux microtubules amène le clivage de ci (facteur de transcription cubitus interruptus) en un répresseur transcriptionnel qui exerce son action dans le noyau. B. Voie active: la liaison du ligand hh (hedgedog) à ptc libère smo de l'action répressive de cette dernière, ce qui entraîne la dissociation du complexe cytoplasmique. ci ne subit pas de clivage et agit comme activateur de la transcription de divers gènes dont ptc lui-même. cos-2: protéine costal-2; fu: sérine-thréonine kinase fused; su(fu): suppresseur de fused. 
ment détectées, tant pour la forme familiale que pour la forme sporadique de ces carcinomes. En outre, comme pour les tumeurs superficielles de la vessie, on observe une perte d'hétérozygotie de marqueurs du locus PTC dans la région $9 q$ dans environ $50 \%$ des carcinomes basocellulaires. La surexpression de GLI-1, ainsi que la présence de mutations dans les gènes $\mathrm{SHH}$ ou Smo peuvent aussi induire un carcinome basocellulaire, suggérant que toute anomalie menant à une surexpression de GLI-1 dans des cellules de la couche basale de la peau peut induire un carcinome $[8,9]$. La capacité de GLI-1 à transformer des cellules en coopération avec le gène $\varepsilon l A$ de l'adénovirus a d'ailleurs confirmé sa nature oncogénique [12]. Des mutations des gènes PTC et Smo ont également été identifiées dans des médulloblastomes et des tumeurs primitives neuroectodermales [8]. Finalement, des souris porteuses d'un allèle inac-

$\mathbf{A}$

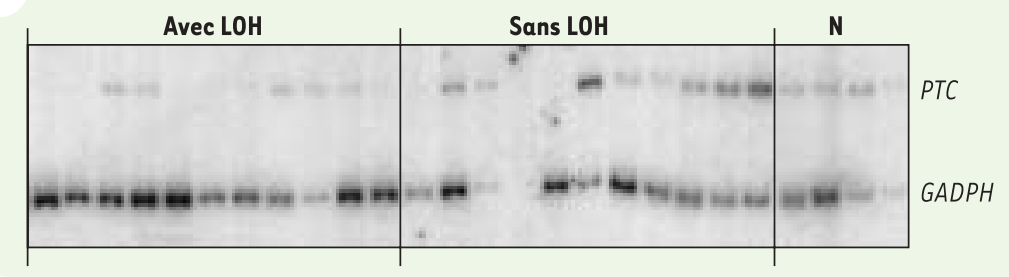

B

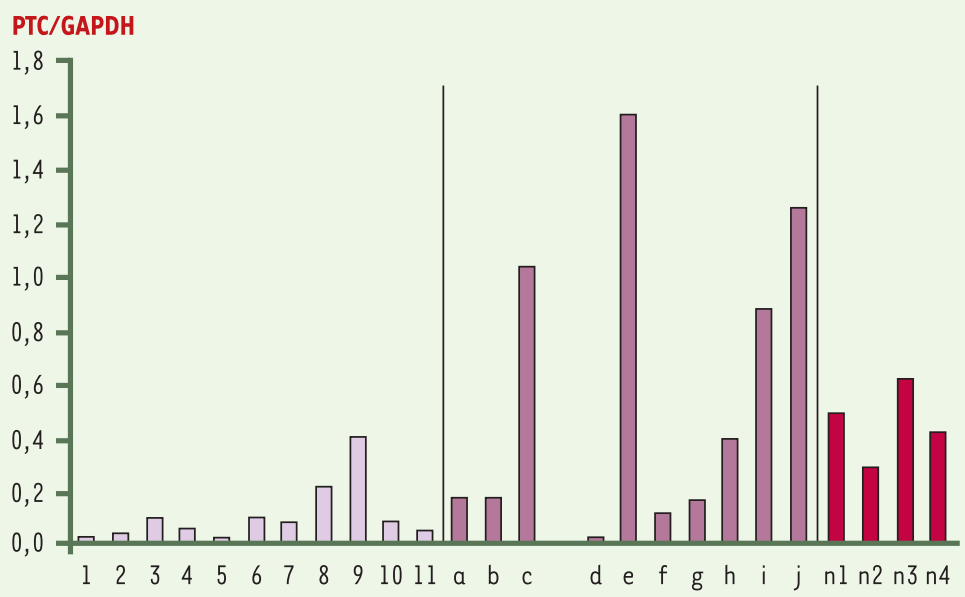

Figure 3. Analyse de l'expression du gène PTC dans des tumeurs vésicales avec perte d'hétérozygotie ( $\mathrm{LOH}$ ) du marqueur PTC, sans $\mathrm{LOH}$ détectée sur le chromosome 9, et dans des urothéliums normaux $(N)$. L'expression du gène a été analysée par la technique de RT-PCR semi-quantitative en présence d'un précurseur radioactif. $A$. Photographie d'un gel d'électrophorèse montrant la migration des fragments amplifiés pour le gène PTC et pour un gène témoin (glycéraldéhyde-3phosphate déhydrogénase (GAPDH)). B. Histogramme montrant le rapport de l'intensité des bandes obtenues pour PTC et GAPDH pour les divers échantillons analysés (d'après [7]). On observe une diminution significative de l'expresion du gène dans les tumeurs ayant perdu une copie de PTC. tivé du gène Ptc développent fréquemment des rhabdo-

\section{Voie de signalisation PTC/SHH}

Il semble donc bien établi que des altérations de la voie PTC/SHH peuvent induire divers cancers. Les délétions fréquentes observées au locus 9 q22 dans des tumeurs de la vessie par diverses équipes, dont la nôtre, amènent naturellement à suspecter le gène PTC comme un élément clé dans la progression conduisant au cancer de la vessie. Afin de vérifier cette hypothèse, la présence de mutations dans le gène PTC a été recherchée dans des tumeurs de la vessie. McGarvey et al. ont détecté des mutations du locus PTC dans deux tumeurs infiltrantes sur 54 tumeurs analysées [15]. Par ailleurs, dans cette étude, l'expression des gènes PTC, Smo et GLI-3 dans l'urothélium normal et dans des lignées cellulaires vésicales a permis de conclure que la voie PTC/SHH pourrait jouer un rôle dans le contrôle de la prolifération urothéliale et contribuerait à la genèse d'au moins un sous-groupe de carcinomes transitionnels de la vessie. En revanche, aucune mutation n'a été trouvée dans trois autres études [1618]. Étant donné que le modèle initialement admis pour un GST suppose l'inactivation des deux allèles du gène, souvent par délétion d'une copie et mutation de l'autre, nous avons séquencé l'ADNc de 15 tumeurs TA ou Tl ayant perdu un allèle de PTC, sans trouver la moindre mutation [7]. La perte d'une copie d'un GST sans altération de l'autre copie ou haploinsuffisance, a été récemment rapportée comme suffisante pour promouvoir le cancer. Diverses études ont, en effet, rapporté I'haploinsuffisance du gène PTEN dans le carcinome rénal à cellules claires [19], du gène SMAD4 dans des cancers gastriques [20], du gène $p 27$ dans divers cancers [21] et du gène PTC lors de l'induction de médulloblastome chez la souris [22]. Nous avons analysé l'expression du gène PTC par la technique de RT-PCR semiquantitative dans 21 tumeurs superficielles de la vessie et 4 échantillons d'urothélium normal humain et avons observé une diminution significative de l'expression de ce gène dans les tumeurs ayant perdu une copie de PTC par rapport 
à son expression dans l'urothélium normal (Figure 3) [7]. Des fluctuations marquées de l'expression de PTC sont aussi constatées dans les tumeurs possédant les deux allèles de PTC suggérant que des mécanismes autre que l'haplo-insuffisance pourraient également contribuer à l'altération de l'expression de ce gène. Des données préliminaires suggèrent la diminution de l'expression d'autres gènes de la voie PTC/SHH.

\section{Conclusions}

L'ensemble de ces données est compatible avec l'hypothèse de la perturbation de la voie de signalisation PTC/SHH au cours des phases précoces de la cancérogenèse vésicale, possiblement en rapport, dans environ la moitié des cas, avec la perte d'une copie du gène PTC. Les carcinomes basocellulaires de la peau et les carcinomes papillaires superficiels de la vessie partageant plusieurs caractéristiques comme une croissance locale, une induction par des carcinogènes environnementaux et un faible potentiel métastatique, il est plausible que des altérations génétiques semblables soient retrouvées dans ces deux types de cancers. Une étude plus approfondie des mécanismes en cause permettra éventuellement l'élaboration de thérapies permettant de ralentir et même de bloquer la prolifération des cellules cancéreuses dans la vessie. $\diamond$

\section{SUMMARY}

The PATCHED/Sonic Hedgehog signalling pathway in superficial bladder cancer

Superficial bladder cancer shows a high frequency of total or partial chromosome 9 losses. Loss of heterozygosity at position $9 q 22.3$ is one of the most frequent and is associated with highly recurrent tumours. The PATCHED gene, ortholog of a gene first described in the drosophila as a segment polarity gene, is located at $9 q 22.3$. It is a member of a signal transduction pathway and a tumour suppressor gene (TSG), involved in basal cell carcinoma. We propose PATCHED as a TSG candidate in superficial bladder cancer. $\diamond$

\section{RÉFÉRENCES}

1. Knowles MA. The genetics of transitional cell carcinoma: progress and potential clinical application. BJU Int 1999; 84: 412-27.

2. Simoneau M, Aboulkassim TO, Larue H, Rousseau F, Fradet $y$. Four tumor suppressor loci on chromosome $9 \mathrm{q}$ in bladder cancer: evidence for two novel candidate regions at $9 q 22.3$ and $9 q 31$. Oncogene 1999; 18: 157-63.

3. Czerniak B, Chaturvedi V, Li $L$, et al. Superimposed histologic and genetic mapping of chromosome 9 in progression of human urinary bladder neoplasia: implications for a genetic model of multistep urothelial carcinogenesis and early detection of urinary bladder cancer. Oncogene 1999. 18: 1185-96.
4. Williams SV, Sibley KD, Davies AM, et al. Molecular genetic analysis of chromosome 9 candidate tumor-suppressor loci in bladder cancer cell lines. Genes Chrom Cancer 2002; $34: 86-96$.

5. Bartlett JMS, Watters AD, Ballantyne SA, Going JJ, Grigor KM, Cooke TG. Is chromosome 9 loss a marker of disease recurrence in transitional cell carcinoma of the urinary bladder? $\mathrm{Br} J$ Cancer 1998; 77: 2193-8.

6. Simoneau $M$, Larue $H$, Aboulkassim TO, Meyer F, Moore L, Fradet $Y$. Chromosome 9 deletions and recurrence of superficial bladder cancer: identification of four regions of prognostic interest. Oncogene 2000; 19: 6317-23.
7. Aboulkassim T0, Larue $H$, Lemieux P, Rousseau F, Fradet $y$. Alteration of the Patched locus in superficial bladder cancer. Oncogene 2003; 22 : 2967-71.

8. Wicking C, Smyth I, Bale A The hedgehog signalling pathway in tumorigenesis and development. Oncogene 1999; 18: 7844-51.

9. Bale AE, Yu KP. The hedgehog pathway and basal cell carcinomas. Hum Mol Genet 2001; 10: 757-62.

10. Goodrich LV, Johnson RL, Milenkovic L, McMahon JA, Scott MP. Conservation of the hedgehog/patched signaling pathway from flies to mice: induction of a mouse patched gene by Hedgehog. Genes Dev 1996; 10: 301-12.
11. Villavicencio $\varepsilon H$, Walterhouse DO, lannaccone PM. The sonic hedgehog-patched-gli pathway in human development and disease. Am J Hum Genet 2000; 67: 1047-54.

12. Altaba A. Gli proteins encode context-dependent positive and negative functions: implications for development and disease. Development 1999; 126: 3205-16.

13. Duman-Scheel M, Weng L, Xin S, Du W. Hedgehog regulates cell growth and proliferation by inducing cyclin D and cyclin $\varepsilon$. Nature 2002; 417: 299-304. 
14. Yoon JW, Kita Y, Frank DJ, et al. Gene expression profiling leads to identification of GLII binding elements in target genes and a role for multiple downstream pathways in GLIl-induced cell transformation. J Biol Chem 2002; 277: 5548-55.

15. McGarvey TW, Maruta Y, Tomaszewski JE, Linnenbach AJ, Malkowicz SB. PTCH gene mutations in invasive transitional cell carcinoma of the bladder. Oncogene 1998; 17: 1167-72.

16. Simoneau $A R$, Spruck $C H$ 3rd, Gonzalez-Zulueta M, et al. Evidence for two tumor suppressor loci associated with proximal chromosome $9 p$ to $q$ and distal chromosome $9 q$ in bladder cancer and the initial screening for GASI and PTC mutations. Cancer Res $1996 ; 56: 5039-43$.
17. Xie J, Johnson RL, Zhang X, et al. Mutations of the PATCHED gene in several types of sporadic extracutaneous tumors. Cancer Res 1997; 57: 2369-72.

18. Ohgaki $K$, Minobe $K$, Kurose $K$, et al. Two target regions of allelic loss on chromosome 9 in urinarybladder cancer. Jpn J Cancer Res 1999 . 90: 957-64.

19. Velickovic M, Delahunt $B$, Mclver B, Grebe SK. Intragenic PTEN/MMACl loss of heterozygosity in conventional (clear-cell) renal cell carcinoma is associated with poor patient prognosis. Mod Pathol 2002; 15 : 479-85.
20. Xu X, Brodie SG, Yang X, et al. Haploid loss of the tumor suppressor Smad4/Dpc4 initiates gastric polyposis and cancer in mice. Oncogene 2000; 19: 1868-74.

21. Philipp-Staheli J, Payne SR, Kemp CJ. p27(Kipl): regulation and function of a haploinsufficient tumor suppressor and its misregulation in cancer. Exp Cell Res 2001; 264: 148-68.
22. Zurawel RH, Allen $\mathrm{C}$, Wechsler-Reya R, Scott MP, Raffel C. Evidence that haploinsufficiency of Ptch leads to medulloblastoma in mice. Genes Chrom Cancer $2000 ; 28: 77-81$.
TIRÉS À PART

H. LaRue

Tarifs d'abonnement pour M/S - 2003

\section{France UE et Suisse Canada USA/Mexique Autres pays}

\begin{tabular}{|c|c|c|c|c|c|}
\hline Particuliers & $\square 118 €$ & $\square 118 €$ & $\square \quad 98$ \$CAN & $\square 98$ \$ US & $\square 118 €$ \\
\hline Institutions & $\square 276 €$ & $\square 320 €$ & $\square 195 \$$ CAN & $\square 195$ \$ US & $\square 338 €$ \\
\hline $\begin{array}{l}\text { Étudiants } \\
\text { sur présentation de ph }\end{array}$ & $\begin{array}{l}\square \quad 50 € \\
\text { otocopie } R^{\circ} / V^{\circ} d\end{array}$ & $\begin{array}{l}\square \quad 50 € \\
\text { te d'étudiant }\end{array}$ & $\square 57$ \$CAN & $\square \quad 57$ \$ US & $50 €$ \\
\hline $\begin{array}{l}\text { Enseignants } \\
\text { sur justificatif }\end{array}$ & $\square \quad 74 €$ & $\square \quad 74 €$ & $\square 98$ \$ CAN & $\square 98$ \$ US & $\square \quad 74 €$ \\
\hline
\end{tabular}

\section{MES COORDONNÉદS}

NOM

PRÉNOM

ADRESSE

CODE POSTAL

PAYS

TEL

ADRESSE Email obligatoire

\section{MON RÈGLEMENT}

$\square$ Par chèque à l'ordre de EDK (France, UE et Suisse)

$\square$ Par chèque à l'ordre de Médecine/Sciences (Canada, USA et Mexique)

$\square$ Par carte de crédit $\square$ Visa $\square$ Eurocard Mastercard $\square$ Amex

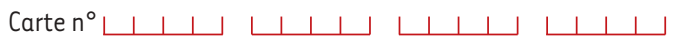

Date d'expiration $\downarrow \downarrow \downarrow \downarrow$ Signature

Pour recevoir une facture, cochez cette case $\square$

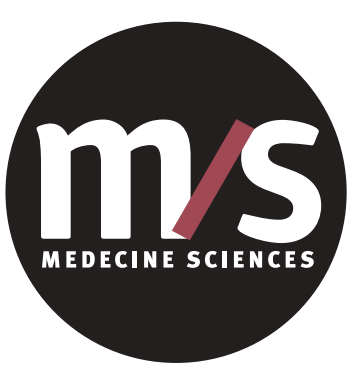

À découper et à renvoyer à :

\section{ATEl}

3, avenue Pierre Kerautret, 93230 Romainville, France.

\section{Médecine/Sciences}

500 , rue Sherbrooke Ouest, bureau 800,

Montréal

Québec H3A 3C6

Canada.

Conformément à la loi Informatique et Libertés du 6 janvier 1978, vous disposez d'un droit d'accès et de rectification des données personelles vous concernant. 